Research Paper

\title{
Establishment and Characterization of an Immortalized Human Hepatic Stellate Cell Line for Applications in Co-Culturing with Immortalized Human Hepatocytes
}

\author{
XiaoPing Pan ${ }^{1,2}$, Yini Wang1,2, XiaoPeng Yu, 1,2, JianZhou Li1,2, Ning Zhou ${ }^{1,2}$, WeiBo Du ${ }^{1,2}$, YanHong \\ Zhang ${ }^{1,2}$, HongCui Cao ${ }^{1,2}$, DanHua Zhu' ${ }^{1,2}, Y u$ Chen ${ }^{1,2}$, LanJuan $\mathrm{Li}^{1,2}$ \\ 1. State Key Laboratory for the Diagnosis and Treatment of Infectious Diseases, First Affiliated Hospital, College of Medicine, Zhejiang \\ University, Hangzhou, 310003, China. \\ 2. Collaborative Innovation Center for the Diagnosis and Treatment of Infectious Diseases, Hangzhou, China.
}

\begin{abstract}
$\square$ Corresponding author: LanJuan Li, M.D. Collaborative Innovation Center for the Diagnosis and Treatment of Infectious Diseases, State Key Laboratory for the Diagnosis and Treatment of Infectious Disease, First Affiliated Hospital, College of Medicine, Zhejiang University, Hangzhou, 310003, China. Tel: 86-571-87236759; Fax: 86-571 87236759; Email: ljli@zju.edu.cn.
\end{abstract}

(c) 2015 Ivyspring International Publisher. Reproduction is permitted for personal, noncommercial use, provided that the article is in whole, unmodified, and properly cited. See http:/ / ivyspring.com/terms for terms and conditions.

Received: 2014.11.06; Accepted: 2015.01.21; Published: 2015.02.08

\begin{abstract}
Background and objective. The liver-specific functions of hepatocytes are improved by co-culturing hepatocytes with primary hepatic stellate cells (HSC). However, primary HSC have a short lifespan in vitro, which is considered a major limitation for their use in various applications. This study aimed to establish immortalized human HSC using the simian virus 40 large T antigen (SV40LT) for applications in co-culturing with hepatocytes and HSC in vitro.

Methods. Primary human HSC were transfected with a recombinant retrovirus containing SV4OLT. The immortalized human HSC were characterized by analyzing their gene expression and functional characteristics. The liver-specific functions of hepatocytes were evaluated in a co-culture system incorporating immortalized human hepatocytes with HSC-Li cells.

Results. The immortalized HSC line, HSC-Li, was obtained after infection with a recombinant retrovirus containing SV4OLT. The HSC-Li cells were longitudinally spindle-like and had numerous fat droplets in their cytoplasm as shown using electron microscopy. Hepatocyte growth factor (HGF), VEGF Receptor 1(Flt-1), collagen type l $\alpha 1$ and l $\alpha 2$ mRNA expression levels were observed in the HSC-Li cells by RT-PCR. Immunofluorescence staining showed that the HSC-Li cells were positive for $\alpha$-smooth muscle actin ( $\alpha$-SMA), platelet-derived growth factor receptor-beta (PDGFR- $\beta$ ), vimentin, and SV4OLT protein expression. The HSC-Li cells produced both HGF and transforming growth factor-betal (TGF- $\beta 1$ ) in a time-dependent manner. Real-time PCR showed that albumin, CYP3A5, CYP2E1, and UGT2B7 mRNA expression generally increased in the co-culture system. The enzymatic activity of CYP1A2 under the co-culture conditions also generally increased as compared to the monoculture of immortalized human hepatocytes.

Conclusions. We successfully established the immortalized human HSC cell line HSC-Li. It has the specific phenotypic and functional characteristics of primary human HSC, which would be a useful tool to develop anti-fibrotic therapies. Co-culturing with the HSC-Li cells improved the liver-specific functions of hepatocytes, which may be valuable and applicable for bioartificial liver systems.
\end{abstract}

Key words: human hepatic stellate cells; simian virus 40 large $\mathrm{T}$ antigen; immortalization; immortalized human hepatocytes; co-culture; bioartificial liver 


\section{Introduction}

Due to the shortage of liver donors for orthotropic liver transplantation, bioartificial liver systems are currently considered as an alternative therapy for liver failure in clinical trials (1). However, bioartificial liver system applications are also limited because suitable liver cell sources are not available. Thus far, immortalized human hepatocytes with indefinite expansion in vitro and allogeneic cells are ideal for bioartificial liver system applications (2-4). However, the functionality and differentiation of these two cell types are lower than the functionality and differentiation of primary human hepatocytes $(5,6)$.

Human hepatic stellate cells (HSC), which are also known as fat-storing cells or Ito cells, are liver non-parenchymal cells that are located in the perisinusoidal tissue space of Disse. In both developing and adult livers, cell-cell interactions, including hepatocytes and stellate cells, have important functions regarding the stability of the liver functions of primary hepatocytes (7). In vitro, the co-culturing of primary hepatocytes with HSC maintains and stabilizes the differentiated phenotype of hepatocytes such as albumin and cytochrome P450 (CYP450) expression (8-10).

However, human HSC are terminally differentiated liver cells, have a limited proliferation capacity and do not passage in vitro for long periods of time. Isolating HSC is also extremely time consuming, laborious and usually produces low yields. Thus, using HSC for bioartificial liver systems or for liver tissue engineering is extremely limited. Cell immortalization is an effective technique to overcome senescence by introducing the simian virus 40 large $\mathrm{T}$ antigen (SV40LT) or human telomerase reverse transcriptase (hTERT) into primary HSC $(11,12)$. Thus, primary human HSC may be exempted from definitive passages by cell immortalization.

In this study, we developed an immortalized human HSC cell line using the retroviral transfer of SV40LT into primary human HSC. We also investigated the enhancement of the liver-specific functions of immortalized human hepatocytes by co-culture with immortalized human HSC.

\section{Materials and Methods}

\section{The preparation of retroviral supernatant containing SV40LT}

A recombinant retroviral vector containing SV40LT was successfully transferred into PT67 retroviral-packaging cells. Virus stocks were collected and stored at an ultra-lower temperature freezer until used as described in our previous study (13).

\section{The isolation and immortalization of human HSCs}

A liver tissue sample was obtained from an adult donor undergoing hepatic resection at the First Affiliated Hospital, School of Medicine, Zhejiang University. This method and the experimental procedures in this study were performed in accordance with the Helsinki Declaration and approved by the ethics committee of the hospital. The patients provided written informed consent to participate in the study. The primary human HSC were isolated from surgical specimens of adult liver donors as previously described $(14,15)$. After $24 \mathrm{~h}$ of culturing, the primary HSC were incubated with $1.5 \mathrm{~mL}$ of retrovirus stocks containing SV40LT and $8 \mu \mathrm{g} / \mathrm{mL}$ polybrene (Aldrich Chemical, USA) at $37^{\circ} \mathrm{C}$ for $6 \mathrm{~h}$ one time a day for 3 days. Three days after the final infection, the primary HSC were selected by adding the neomycin analog G418 (Sigma, USA) at a final concentration of $450 \mu \mathrm{g} / \mathrm{mL}$. One of the transferred HSC, called HSC-Li, was specifically selected due to its phenotypic and functional characteristics and was continuously expanded by passaging in vitro.

\section{Electron microscopy examination}

The ultra microstructure of immortalized human HSC was observed under an electron microscope. For scanning electron analysis, the HSC-Li cells were prepared using standard techniques and were observed using a Cambridge Stereoscan 260 SEM. For transmission electron analysis, the HSC-Li cells were also prepared using standard techniques and were examined using a Philips TECNAI 10 TEM. The analysis procedure to determine the ultra microstructure of the immortalized human HSC was previously described in detail $(13,16)$.

\section{Reverse transcription PCR analysis}

The mRNA of the immortalized human HSC was extracted from T25 flask cultures using TRIzol according to the manufacturer's instructions. RT-PCR was performed using a one-step RT-PCR reagent kit according to the manufacturer's instructions (Invitrogen, USA). The PCR products were visualized using a Bio-Rad image instrument under ultraviolet light. The primers and annealing temperatures for the PCR amplification of SV40LT, VEGF Receptor 1(Flt-1), HGF, collagen type I $\alpha 1$, collagen type I $\alpha$ and human $\beta$-actin mRNAs were previously described $(13,17)$.

\section{Immunofluorescence staining}

The immortalized human HSC were inoculated onto overlaps (Fisher, USA) in six-well plates. After $24 \mathrm{~h}$ of culture, the immortalized HSC attached onto the overlaps were washed twice with PBS. The over- 
laps were transferred and incubated in cold acetone for $10 \mathrm{~min}$ of fixing. Next, the overlaps were washed twice with PBS, permeabilized with PBS containing $0.1 \%$ Triton X-100 for $15 \mathrm{~min}$, and then washed twice with PBS. Then, the overlaps were incubated in blocking solution at $37^{\circ} \mathrm{C}$ for $30 \mathrm{~min}$ and PBS containing 3\% BAS with primary antibody for $120 \mathrm{~min}$. After washing with PBS, the overlaps were incubated with a secondary antibody in PBS containing 3\% BAS for $60 \mathrm{~min}$. The overlaps were finally washed twice with PBS, and DAPI (ENZO, USA) was used for cell nucleus staining. The overlaps were visualized by fluorescence microscopy (Olympus, Japan). The primary antibodies included anti-human vimentin antibody (R\&D Systems, USA), anti-human a-smooth muscle actin (a-SMA) antibody (R\&D Systems, USA), platelet-derived growth factor receptor-beta (PDGFR- $\beta$ ) (Santa Cruz, USA) and mouse monoclonal anti-SV40LT antibody (Santa Cruz, USA). The secondary antibodies included FITC fluorescent goat anti-mouse antibody and donkey anti-rabbit antibody (Abcam, USA).

\section{Low-density lipoprotein (LDL) uptake assay}

The HSC-Li cells $\left(1.0 \times 10^{5}\right.$ cells/well $)$ were inoculated into six-well plates with DMEM media containing 10\% FBS. After $24 \mathrm{~h}$ of incubation, these cells were incubated with the same media supplemented with $10 \mu \mathrm{g} / \mathrm{mL}$ DiI-LDL (fluorescent LDL; Molecular Probes, Invitrogen, USA) for another $24 \mathrm{~h}$ at $37^{\circ} \mathrm{C}$. The HSC-Li cells were washed twice with cold PBS, and DiI-LDL uptake was observed under a fluorescence microscope (Olympus, $1 \times 81$, Japan).

\section{Hepatocyte growth factor (HGF) and transforming growth factor-betal (TGF- $\beta 1$ ) production in HSC-Li cells}

To measure HGF and TGF- $\beta 1$ secretion in the HSC-Li cells, we inoculated $1.0 \times 10^{6}$ of HSC-Li cells into $90 \mathrm{~mm}$ dishes. After $24 \mathrm{~h}$ of culture, the cells were cultured with DMEM media supplemented with low amounts of FBS for eleven days. The culture supernatant of the HSC-Li cells was collected daily and assayed using human HGF and TGF- $\beta 1$ ELISA kit (R\&D Systems, USA) according to the manufacturer's manual.

\section{Growth curve of immortalized human HSC}

To plot the growth curve of the HSC-Li cells, we inoculated the HSC-Li cells into six-well plates at an initial density of $2.0 \times 10^{4}$ cells/well. The cell numbers were calculated once a day for 11 days using trypan blue exclusion tests. Three wells were included in each cell count.

\section{Tumorigenicity analysis}

This study was performed in strict accordance with the recommendations in the Guide for the Care and Use of Laboratory Animals of the National Institutes of Health. The protocol was approved by the Committee on the Ethics of Animal Experiments of School of Medicine, Zhejiang University (Permit Number: ZJU201308-1-01-086). All surgery was carried out under sodium pentobarbital anesthesia in order to minimize suffering.

To analyze the potential tumorigenicity of the immortalized HSC, we subcutaneously injected $2.0 \times 10^{6}$ HSC-Li cells into 8-week-old nude mice. Eight mice were included in the study. As positive controls, $2.0 \times 10^{6} \mathrm{HepG} 2$ cells were also injected into eight nude mice $(N=8)$. All of the mice were examined for tumor formation weekly for three months.

\section{Co-culture of immortalized human hepatocytes with HSC-Li cells}

We investigated whether the co-culture with HSC-Li cells improve the liver-specific functions of the immortalized human hepatocytes, HepLi5 cells (15). As shown in Figure 1, direct (mixed) and indirect (Transwell) co-cultures were performed to analyze the effects of co-culturing with HSC-Li cells on the liver-specific functions of immortalized human hepatocytes in comparison with the monoculture (control) group.
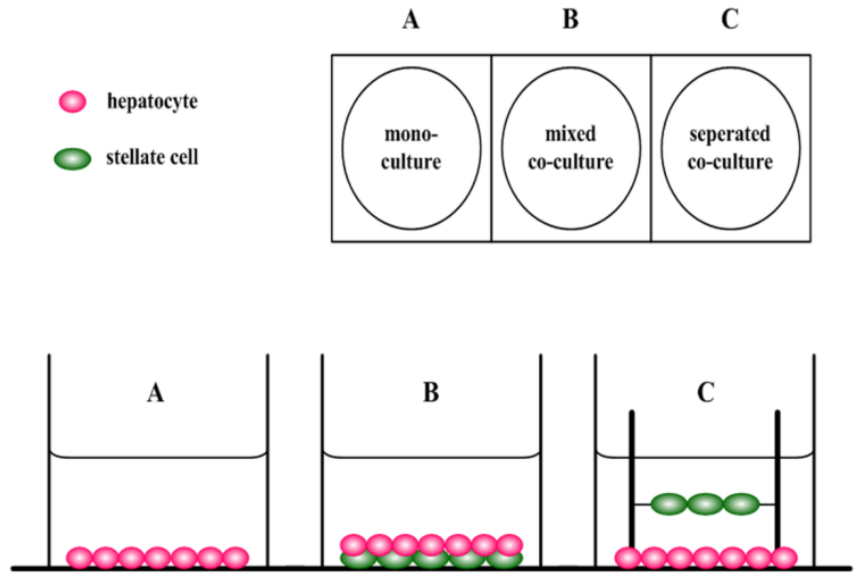

Figure 1. Co-culture systems used in the experiments. A. Hepatocyte monoculture. HepLi5 cells were inoculated into the lower layer of a Transwell dish. B. Mixed co-culture. HepLi5 and Dil-HSC-Li cells were all inoculated into the lower layer of a Transwell dish. C. Separated co-culture. HepLi5 and Dil-HSC-Li cells were inoculated into the lower and upper layers of a Transwell dish, respectively.

To easily sort HepLi5 cells from the mixed co-culture, we used 1,1'-dioctadecyl-3,3,3',3'-tetramethylindocarbocyanine perchlorate (DiI), a fluorescent dye that diffuses within cell membranes, to mark the HSC-Li cells. $2.0 \times 10^{5} \mathrm{HepLi5}$ cells and $1.0 \times 10^{5}$ 
DiI-HSC-Li cells were mixed and inoculated into the lower layer of dishes in a mixed co-culture. In a Transwell co-culture system, $2.0 \times 10^{5} \mathrm{HepLi5}$ cells and $1.0 \times 10^{5}$ DiI-HSC-Li cells were inoculated into the lower and upper layers of the dishes, respectively, without any cell-cell contact using a culture insert (pore size: $3.0 \mu \mathrm{m}$; Millipore, Billerica, MA, USA). In the monoculture (control) group, $2.0 \times 10^{5} \mathrm{HepLi5}$ cells were inoculated into the lower layer of the dishes. The supernatant and cells were collected from the different groups after 24, 48, and $72 \mathrm{~h}$.

\section{Separation of Dil-positive and Dil-negative cells in cocultures by fluorescence activated cell sorting (FACS)}

After the culture period, the cells from the mixed co-cultures were trypsinized, counted using trypan blue, and resuspended in PBS. To get single cell suspension of HepLi5 cells, Cells were sorted using BD Aria II Cell Sorting System (BD, USA) into Dil-positive or DiI-negative cells, focusing on the highest possible purity of HepLi5 cells.

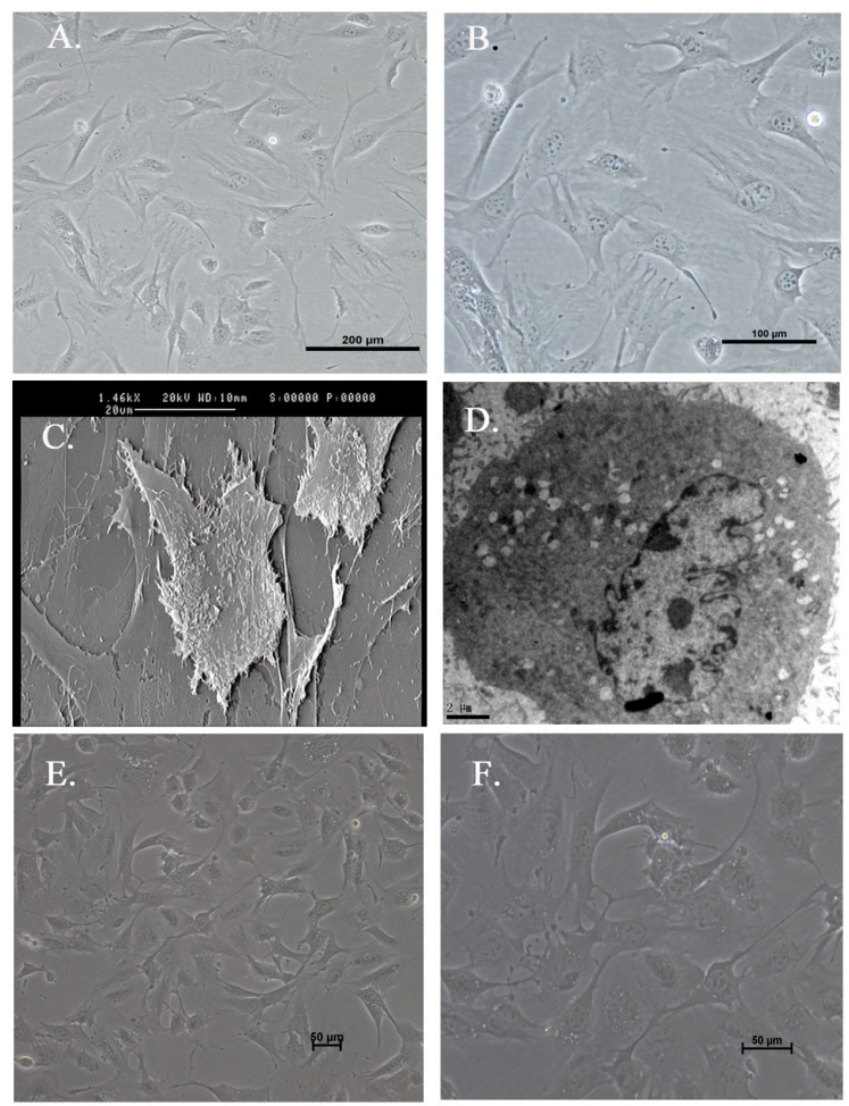

Figure 2. Morphological features of immortalized human HSC. A and $B$. The HSC-Li cells at 30 passages were generally elongated and longitudinally spindle-like as observed under a contrast phase microscope. Original magnification: $\times 100$ and $\times 200$, respectively. C and D. HSC-Li cells were longitudinally spindle-like and had numerous fat droplets in their cytoplasm as observed under an electron microscope. Original magnification: $\times 1460$ and $\times 6200$, respectively. $E$ and $F$. The morphology of HSC-Li cells at 100 passages were also showed by contrast phase microscope. Original magnification: $\times 100$ and $\times 200$, respectively.

\section{CYP450 enzymatic activity}

Meanwhile, the enzymatic activity of CPY450 in HepLi5 cells from different groups was assessed by measuring luciferase activity using a P450-GloTM CYP1A2 assay (V8422; Promega, USA) according to the manufacturer's instructions. In brief, HepLi5 cells from different groups were incubated at $37^{\circ} \mathrm{C}$ in Krebs-Henseleit buffer supplemented with luciferin-1A2. After $1 \mathrm{~h}$ of incubation, $50 \mu \mathrm{l}$ of buffer from each well was transferred into a 96-well opaque white plate and mixed with $50 \mu \mathrm{l}$ of luciferin detection reagent. After $20 \mathrm{~min}$ of incubation at room temperature, luminescence was assayed with a multimode reader (DTX880; Beckman Coulter, USA).

\section{Real-time quantitative RT-PCR}

The total cellular RNA of the HepLi5 cells from different groups was extracted using an RNeasy ${ }^{\circledR}$ Plus Mini Kit (Qiagen, USA). cDNA synthesis was performed using a reverse transcription kit (Promega, USA) in accordance with the manufacturer's protocol. The mRNA expression levels of albumin, CYP3A5, CYP2E1, and UGT2B7 in HepLi5 cells were quantified using real-time RT-PCR with a SYBR Premix Ex Kit (Takara Biotech Co. Ltd., Dalian, China) and with an ABI 7500 Detector (Applied Biosystems). The real-time PCR amplification parameters and the primer sequences were described in detail in our previous study (15). The relative mRNA expression levels were analyzed using the Delta-Delta Ct method and normalized to GAPDH.

\section{Statistical analysis}

The quantitative values are presented as the mean \pm SD. All data analyses were performed using SPSS 15.0 software (Chicago, IL, USA). Student's t-tests were performed to analyze different significances in the mean values. A $P$ value $<0.05$ was considered statistically significant.

\section{Results}

\section{The establishment of immortalized human HSCs}

Three immortalized human HSC lines were successfully established by transferring primary human HSC with a retroviral supernatant containing SV40LT. The immortalized human HSC cell line, HSC-Li, was investigated in this study. Under a contrast phase microscope, the HSC-Li cells were generally elongated and longitudinally spindle-like and exhibited hill-and-valley morphology (Fig. 2A and B). Electron microscopy examination showed that the HSC-Li cells were longitudinally spindle-like and had many fat droplets in their cytoplasm (Fig. 2C and D). 
Moreover, the morphology of HSC-Li cells at 100 passages were also showed by contrast phase microscope (Fig. 2E and F). The results indicated that HSC-Li cells were cultured for more than 100 passages without any crisis. The RT-PCR and immunofluorescence staining showed that the HSC-Li cells were positive for SV40LT gene and protein expression (Figs. 3 and 4A). All of these results indicate that the cells were immortalized.

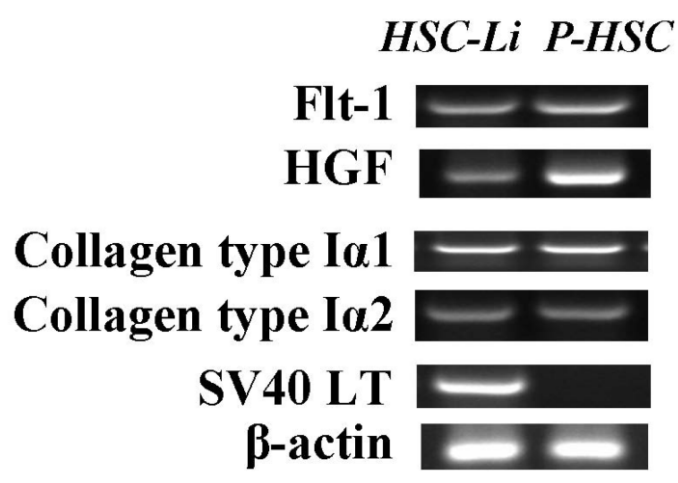

Figure 3. The expression of HSC markers and SV40LT in HSC-Li cells. The HSC-Li cells expressed the following genes: Flt-1, HGF, collagen type $\mid \alpha 1$, collagens type l $\alpha 2$, and SV40LT. Primary hepatic stellate cells (P-HSC) served as the control.

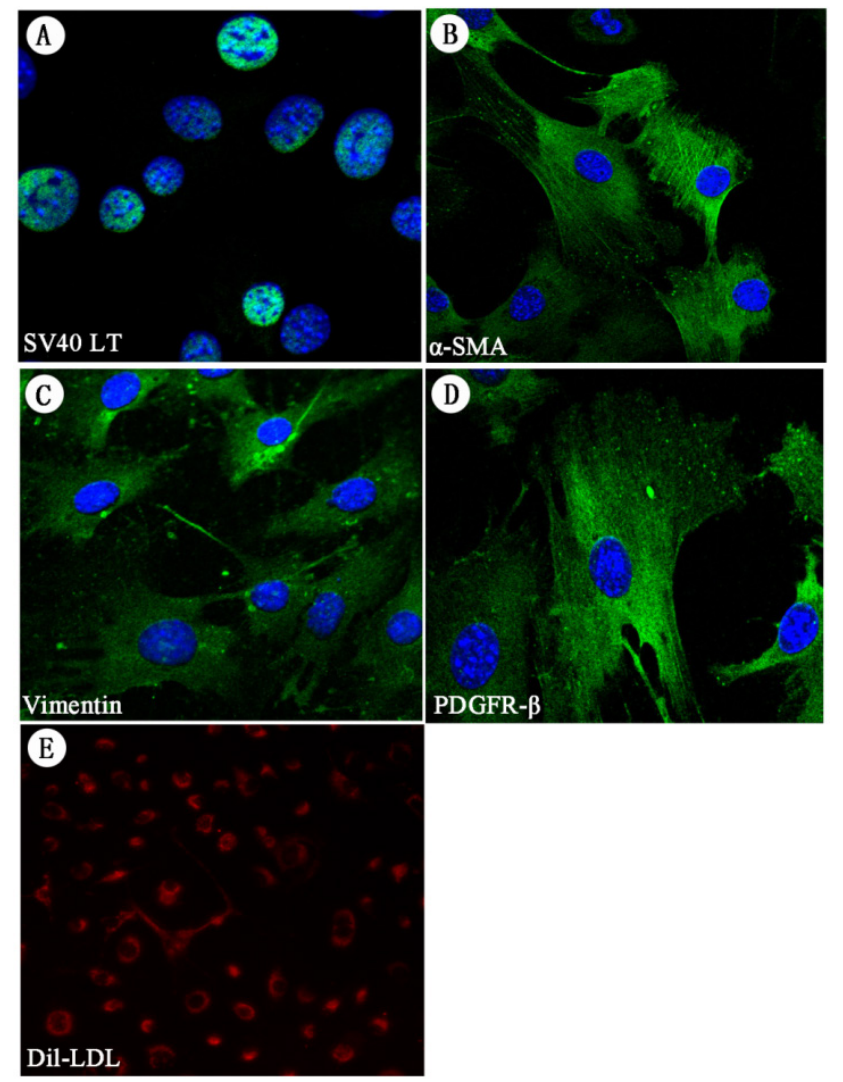

Figure 4. Immunofluorescence staining of HSC-Li cells and Dil-LDL uptake. A. The expression of SV40LT proteins was positive in the HSC-Li cells. It was localized in the nucleus of HSC-Li cells. Cells were costained with DAPI to identify nuclei. Original magnification: $\times 400$. B-D. The expression of $\alpha-S M A$, vimentin, and PDGFR- $\beta$ proteins was also positive in the HSC-Li cells. Cells were costained with DAPI to identify nuclei. Original magnification: $\times 400$. E.The HSC-Li cells strongly endocytosed LDL after $24 \mathrm{~h}$ of culture with Dil-LDL. Original magnification: $\times 200$.
The gene expression of HSC markers in HSC-Li cells

The gene expression of HSC markers was detected by RT-PCR in HSC-Li cells at 30 passages. The RT-PCR showed that the HSC-Li cells expressed Flt-1, collagen type I $\alpha 1$, and collagen type I $\alpha 2$. HGF expression was particularly abundant in HSC-Li cells (Fig. 3).

\section{Immunofluorescence staining for the protein expression of HSC markers in HSC-Li cells}

We also detected the protein expression of intermediate filaments, including a-SMA and vimentin in HSC-Li cells. The immunofluorescence staining showed that HSC-Li cells strongly expressed a-SMA, vimentin, and PDGFR- $\beta$ proteins (Fig. 4B-D).

\section{Dil-LDL uptake in HSC-Li cells}

The HSC-Li cells were incubated with DiI-LDL for $24 \mathrm{~h}$. Fluorescence microscopy showed that the HSC-Li cells strongly endocytosed DiI-LDL (Fig. 4E).

\section{HGF and TGF- $\beta 1$ production in HSC-Li cells}

The HGF and TGF- $\beta 1$ concentration in the supernatant of the HSC-Li cells was measured by ELISA. The concentrations were $128.33 \pm 8.02$, $411.0 \pm 9.85$ and $1536.00 \pm 44.36 \mathrm{pg} / \mathrm{mL}$ after $24 \mathrm{~h}, 72 \mathrm{~h}$, and 11 days of culture, respectively (Fig. 5A). These results indicate that the HSC-Li cells produced HGF in a time-dependent manner. Meanwhile, the levels of TGF- $\beta 1$ secretion were significantly increased during the period of culture (Fig. 5A). These results also indicate that the HSC-Li cells produced TGF- $\beta 1$ in a time-dependent manner.

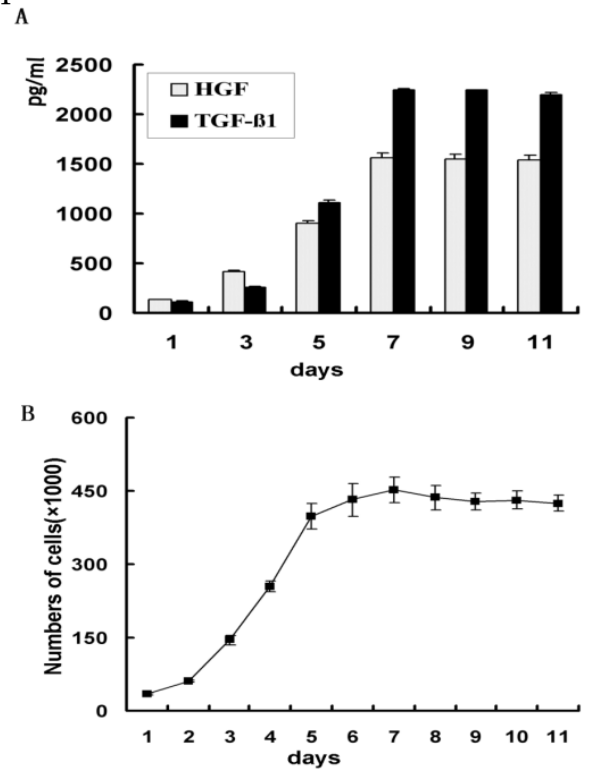

Figure 5. HGF and TGF- $\beta 1$ production in HSC-Li cells and growth curve of HSC-Li cells. A. The HSC-Li cells produced both HGF and TGF- $\beta 1$ in a time-dependent manner. B. Growth curve of HSC-Li cells. 


\section{Growth curve}

The growth curve of the HSC-Li cells was drawn with the culture time as the abscissa and with the cell numbers as the ordinate. The HSC-Li cells proliferated significantly from $2.0 \times 10^{4}$ cells/well to $42.5 \pm 1.6$ $\times 10^{4}$ cells/well throughout all observation periods (Fig. 5B). The HSC-Li cells proliferated with a doubling time of $8 \mathrm{~h}$. Moreover, the HSC-Li cell line was cultured in DMEM with only 1\% FBS. However, it was not able to grow under serum free conditions.

\section{Tumorigenicity analysis}

Regarding tumorigenicity analysis, $2.0 \times 10^{6}$ HSC-Li cells were subcutaneously injected into 8 -week-old nude mice. No tumors formed during the three-month observation period. However, tumors were observed in the nude mice approximately three weeks after the injection of $2.0 \times 10^{6}$ HepG2 cells.

\section{FACS of co-cultured cells}

The sorting strategy aimed at highest possible purity of HepLi5 cells to exclude contamination with HSC-Li cells-derived RNA used in the quantitative RT-PCR. A representative example for FACS after $72 \mathrm{~h}$-coculture is shown in Fig.6. The viable cells of HepLi5 and HSC-Li cells were gated according to forward and side scatter properties, which were located in p3 and used as a negative control (Fig.6A). The Dil-HSC-Li cells were gated in p4 and used as a positive control (Fig.6B). The sample of HepLi5 and DiI-HSC-Li cells from72h after the mixed co-culture were located in p3 and in p4, respectively. The cells from p3 were selectively sorted using FACS (Fig.6C). The sorted cells from p3 were analyzed by Flow cytometry again. The results showed that the sorted cells were all located in p3, indicating that HepLi5 cells were successfully sorted from the mixed co-culture of HepLi5 and DiI-HSC-Li cells (Fig.6D).

\section{The effects of co-culture with HSC-Li cells on the hepatocyte-specific functions of immortalized human hepatocytes}

To optimize the degree of heterotypic cell-cell interactions between the HepLi5 and HSC-Li cells, we varied the ratios of these cells from 5:1 to 1:5. The 2:1 ratio (HepLi5: HSC-Li cells) in the co-culture showed the best improvement in CYP1A2 activity and in albumin expression in the HepLi5 cells (data not shown). Thus, this ratio was used in our subsequent experiments. We used real-time quantitative PCR to assay the effect of co-culturing with the HSC-Li cells on the liver-specific gene expression of HepLi5 cells between monoculture and co-culture. Real-time PCR showed that the albumin, CYP3A5, CYP2E1, and UGT2B7 mRNA expression levels in the HepLi5 cells in separated co-culture and in mixed co-culture were generally higher than the expression levels in a monoculture of HepLi5 cells (Fig. 7A-D).

We also compared the CYP1A2 activity levels in the HepLi5 cells between a monoculture of HepLi5 cells and a co-culture of HepLi5 cells with the HSC-Li cells. The CYP1A2 activity levels in the HepLi5 cells in a separated co-culture and in a mixed co-culture were generally higher than that in a monoculture of HepLi5 cells (Fig. 7E).
A

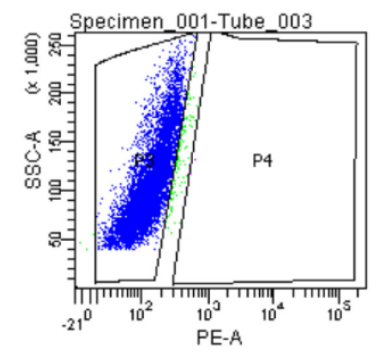

C

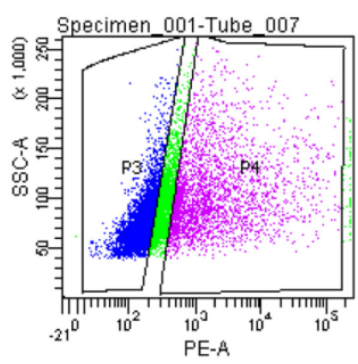

B

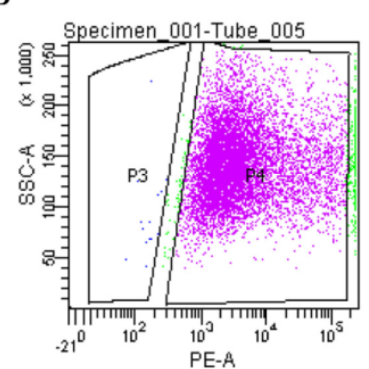

D

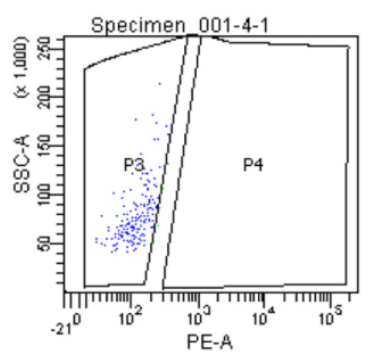

Figure 6. FACS of co-cultured cells. A. The viable cells of HepLi5 and HSC-Li cells were gated, which were located in $\mathrm{p} 3$ and used as a negative control. B. The Dil-HSC-Li cells were gated in $\mathrm{p} 4$ and used as a positive control. C. The sample of HepLi5 and Dil-HSC-Li cells from $72 \mathrm{~h}$ after the mixed co-culture were located in $\mathrm{p} 3$ and in $\mathrm{p} 4$, respectively. The cells from $\mathrm{p} 3$ were selectively sorted using FACS. D. The results of Flow cytometry analysis showed that the sorted cells were all located in p3, indicating that the HepLi5 cells were successfully sorted from the mixed co-culture.

\section{Discussion}

In the present study, we established immortalized human HSC cell line by transfecting a recombinant retrovirus containing SV40LT into primary human HSC. HSC-Li cells, which were derived from one of the immortalized human HSCs, possessed morphological features of HSC. Specifically, the HSC-Li cells expressed HSC markers such as collagen type I $\alpha 1$, HGF, PDGFR- $\beta$ and collagen type I $\alpha 2$ as well as $\alpha-S M A$ and TGF- $\beta 1$. The results of this study demonstrate that co-culturing with the HSC-Li cells significantly improved the liver-specific gene expression and functions of the HepLi5 cells.

HSC, which are the nearest neighbors of hepatocytes, has an important function in the proliferation and maintenance of hepatocyte differentiation. However, these cells have a finite life span. Te- 
lomere shortening controls the entry of HSC into senescence. The functional expression of hTERT stabilizes telomere length and extends the lifespan of primary human HSC $(12,18)$. SV40LT expression enables rat primary stellate cells to surpass replicative senescence and to extend the lifetime of rat primary stellate cells (11). In this study, the HSC-Li cells were established by transferring SV40LT into primary human HSC. The HSC-Li cells were longitudinally spindle-like and had numerous fat droplets in their cytoplasm as observed under an electron microscope. SV40LT gene and protein expression were strongly positive in the HSC-Li cells based on RT-PCR and immunofluorescence staining. Moreover, HSC-Li cells possessed preliminarily morphological features of HSC after in vitro 100 passages. All of these results demonstrate that the HSC-Li cells were immortalized human HSC.

The immunofluorescence staining indicated that the HSC-Li cells strongly expressed a-SMA and

$$
\text { A }
$$
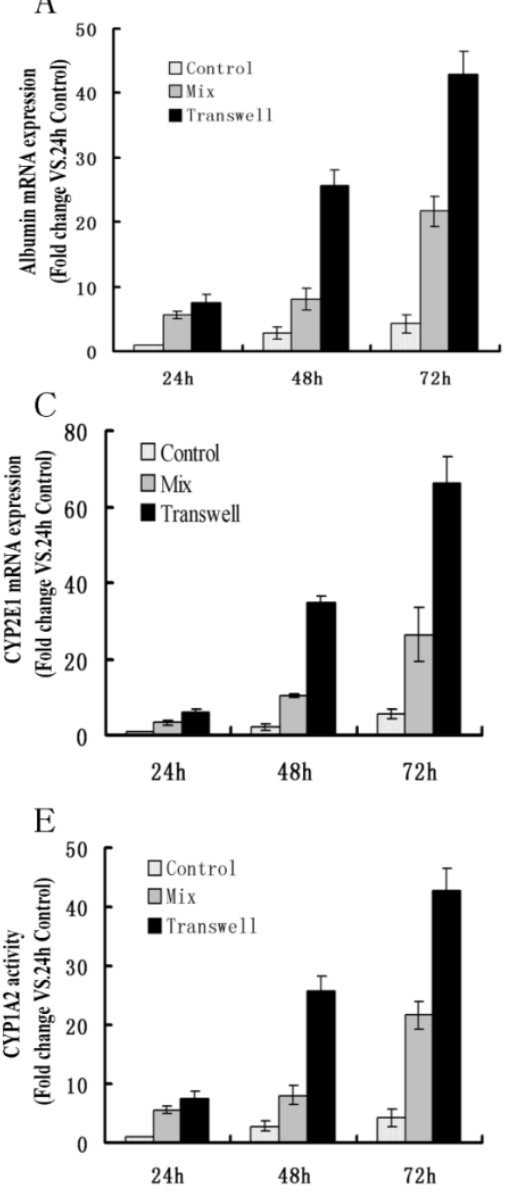

Figure 7. The effects of co-culturing with HSC-Li cells on hepatocyte-specific functions of HepLi5 cells. A-D. The ratio of gene expression levels in HepLi5 cells between monolayer culturing and co-culturing as determined using real-time RT-PCR. The relative expression levels were determined by the Delta-Delta $\mathrm{Ct}$ method and normalized to GAPDH. The mRNA expression levels of albumin, CYP3A5, CYP2E1, and UGT2B7 in HepLi5 cells in mixed co-culture and in separated co-culture were generally higher than the levels in monolayer-cultured HepLi5 cells alone. (E). The CYPIA2 activity of HepLi5 cells in separated co-culture and in mixed co-culture was generally higher than in a monoculture of $\mathrm{HepLi5}$ cells.
PDGFR- $\beta$, which are characteristics of HSC. And the HSC-Li cells produced TGF- $\beta 1$ in a time-dependent manner. Especially, the HSC-Li cells expressed specific HSC markers such as collagen type I $\alpha 1$, collagen type I $\alpha 2$ and PDGFR- $\beta$ as well as $\alpha-S M A$ and TGF- $\beta 1$. These findings suggested that immortalized activated HSC line HSC-Li would be a useful tool to develop anti-fibrotic therapies.

In addition, the HSC-Li cells also expressed HGF and Flt-1 and strongly endocytosed LDL. And the HSC-Li cells produced HGF in a time-dependent manner. Moreover, the HSC-Li cells could be cultured in DMEM with lower serum conditions. And the HSC-Li cells were not tumorigenic throughout 3 months of observation. Thus, the HSC-Li cells may be useful for tissue engineering and for the co-culturing of hepatocytes and non-parenchymal liver cells.

The communication between primary hepatocytes and non-parenchymal liver cells has an important function in maintaining the liver-specific function of primary hepatocytes $(19,20)$. HSC are considered to have a critical function in regulating primary rat hepatocyte proliferation or in preserving several key aspects of differentiated hepatocyte function in vitro $(10,21)$. In this study, we used HSC-Li cells to analyze the effects of co-culturing on the liver-specific functions of immortalized human hepatocytes. The real-time PCR results indicated that the mRNA expression levels of albumin, CYP3A5, CYP2E1, and UGT2B7 in the HepLi5 cells were generally upregulated after co-culturing with the HSC-Li cells. Moreover, human CYP450, which is a marker of liver-specific biotransformation functions, has an important function in the metabolism of most drugs. The CYP1A2 activity level in the HepLi5 cells also generally improved after co-culturing with HSC-Li cells. All of these results demonstrate that both the transcriptional levels of liver-specific genes and CYP450 activities were enhanced when the HepLi5 cells were co-cultured with the HSC-Li cells. The obtained results were consistent with the results of a previous study, which found that heterotypic interactions 
are important for enhanced hepatocyte functions (17, 22). The results of the present study also demonstrated that the liver functions of hepatocytes in separated co-culture were considerably higher than the functions of hepatocytes in mixed co-culture. These similar results were proven by another study that demonstrated that the DNA synthesis of primary rat hepatocytes in mixed co-culture is considerably lower than the level during separated co-culture conditions (21). These results suggest that the cell-cell indirect contact between hepatocytes and HSC significantly enhance hepatocyte functions.

HGF is a potential endogenous mitogen for hepatocytes. This study demonstrated that the HSC-Li cells produced HGF in a time-dependent manner, indicating that HGF enhances hepatocyte functions. Moreover, the liver-specific functions of hepatocytes in separated co-cultures were considerably higher than these hepatocyte functions in a mixed co-culture. We speculate that soluble factors secreted by HSC-Li cells are involved in this co-culture system. However, the mechanisms by which the liver functions of hepatocytes are upregulated by HSC have not been elucidated. Thus, further investigations are required to determine the underlying mechanisms.

In conclusion, the HSC-Li cells were successfully established by introducing SV40LT into primary human HSC. The HSC-Li cells exhibited the morphological features of HSC and expressed HSC markers. The hepatocyte function in the HepLi5 cells was significantly improved by co-culturing with the HSC-Li cells. This co-culture system is a promising approach to enhance the liver-specific functions of hepatocytes for potential applications in bioartificial liver systems.

\section{Acknowledgements}

This work was supported by the Chinese High Tech Research \& Development (863) Program (No.2013AA020102 and 2012AA020204), Science Fund for Creative Research Groups of the National Natural Science Foundation of China (No.81121002), Fundamental Research Funds for the Central Universities (No.2014XZZX008 and 2014FZA7010), Zhejiang Health Science Foundation (2009A076) and Technology Group Project for Infectious Disease Control of Zhejiang Province (2009R50041).

\section{Author Contributions}

Conceived and designed the experiments: LJ and $\mathrm{XP}$. Performed the experiments: XP, XP, JZ and YH. Analyzed the data: $X P$ and $W B$. Contributed reagents/materials/analysis tools: $\mathrm{XP}, \mathrm{XP}, \mathrm{YN}, \mathrm{HC}, \mathrm{ZN}$, $\mathrm{DH}$ and $Y C$. Wrote the paper: XP.

\section{Competing Interests}

The authors have declared that no competing interest exists.

\section{References}

1. Carpentier B, Gautier A, Legallais C. Artificial and bioartificial liver devices: present and future. Gut. 2009; 58:1690-702.

2. Ding YT, Shi XL. Bioartificial liver devices: Perspectives on the state of the art. Front Med. 2011;5:15-9.

3. Hoekstra R, Chamuleau RA. Recent developments on human cell lines for the bioartificial liver. Int J Artif Organs. 2002;25:182-91.

4. Streetz KL. Bio-artificial liver devices--tentative, but promising progress. J Hepatol. 2008; 48:189-91.

5. Poyck PP, van Wijk AC, van der Hoeven TV, et al. Evaluation of a new immortalized human fetal liver cell line (cBAL111) for application in bioartificial liver. J Hepatol. 2008;48:266-75.

6. Totsugawa T, Yong C, Rivas-Carrillo JD, et al. Survival of liver failure pigs by transplantation of reversibly immortalized human hepatocytes with Tamoxifen-mediated self-recombination. J Hepatol. 2007; 47:74-82.

7. Bhatia SN, Balis UJ, Yarmush ML, et al.Effect of cell-cell interactions in preservation of cellular phenotype: cocultivation of hepatocytes and nonparenchymal cells. Faseb J. 1999;13:1883-900.

8. Rojkind M, Novikoff PM, Greenwel P, et al. Characterization and functional studies on rat liver fat-storing cell line and freshly isolated hepatocyte coculture system. Am J Pathol. 1995;146:1508-20.

9. Abu-Absi SF, Hansen LK, Hu WS. Three-dimensional co-culture of hepatocytes and stellate cells. Cytotechnology. 2004;45:125- 40.

10. Thomas RJ, Bhandari R, Barrett DA, et al. The effect of three-dimensional co-culture of hepatocytes and hepatic stellate cells on key hepatocyte functions in vitro. Cells Tissues Organs. 2005; 181:67-79.

11. Vogel S, Piantedosi R, Frank J, et al. An immortalized rat liver stellate cell line (HSC-T6): a new cell model for the study of retinoid metabolism in vitro. J Lipid Res. 2000;41:882-93.

12. Schnabl B, Choi YH, Olsen JC, et al. Immortal activated human hepatic stellate cells generated by ectopic telomerase expression. Lab Invest. 2002;82:323-33.

13. Pan X, Du W, Yu X, et al. Establishment and characterization of immortalized porcine hepatocytes for the study of hepatocyte xenotransplantation. Transplant Proc. 2010;42:1899-906.

14. Friedman SL, Rockey DC, McGuire RF, et al. Isolated hepatic lipocytes and Kupffer cells from normal human liver: morphological and functional characteristics in primary culture. Hepatology. 1992;15:234-43.

15. Pan X, Li J, Du W, et al. Establishment and characterization of immortalized human hepatocyte cell line for applications in bioartificial livers. Biotechnol Lett. 2012; 34:2183-90.

16. Lazaro CA, Croager EJ, Mitchell C, et al. Establishment, characterization, and long-term maintenance of cultures of human fetal hepatocytes. Hepatology. 2003;38:1095-106.

17. Watanabe T, Shibata N, Westerman KA, et al. Establishment of immortalized human hepatic stellate scavenger cells to develop bioartificial livers. Transplantation. 2003;75:1873-80.

18. Waki K, Anno K, Ono T, et al. Establishment of functional telomerase immortalized human hepatocytes and a hepatic stellate cell line for telomere-targeting anticancer drug development. Cancer Sci. 2010;101:1678-85.

19. Begue JM, Guguen-Guillouzo C, Pasdeloup N, et al. Prolonged maintenance of active cytochrome P-450 in adult rat hepatocytes co-cultured with another liver cell type. Hepatology. 1984; 4:839-42.

20. Lu HF, Chua KN, Zhang PC, et al. Three-dimensional co-culture of rat hepatocyte spheroids and NIH/3T3 fibroblasts enhances hepatocyte functional maintenance. Acta Biomater. 2005; 1:399-410.

21. Uyama N, Shimahara Y, Kawada N, et al. Regulation of cultured rat hepatocyte proliferation by stellate cells. J Hepatol. 2002;36:590-9.

22. Okamoto M, Ishida Y, Keogh A, et al. Evaluation of the function of primary human hepatocytes co-cultured with the human hepatic stellate cell (HSC) line LI90. Int J Artif Organs. 1998;21:353-9. 